\title{
Tribute to Prof. Jacques Demongeot: conversations at the National Bar
}

\author{
Eric Goles ${ }^{1}$
}

Published online: 3 January 2020

(C) Springer Nature B.V. 2019

The last time Jacques Demongeot came to Santiago, just a couple of months ago, while we were eating a cazuela (chilean stew) at the National Bar, he told me that one of his aunts had been, in Rome, the superior mother of a congregation whose name I don't remember. Of course, he added, he was a Catholic by family and, just in case, he always carried a relic. Before I, supposedly unbelieving (I wasn't), asked him for some proof, he took out of his backpack a little box with holy bones.

The majority, those who hardly know him, after this staging, would quickly finish eating and leave - like we say in spanish: "si te he visto no me acuerdo" (if I've seen you I don't remember). But not me, nor any of the privileged ones who have allowed ourselves to be enveloped by the magic of the marvelous (and very scarce) person that he is: between the joyful excess of Rabelais and the sharp certainties of Descartes. And always from the truth, with the necessary excess for, as the poet William Blake affirmed, reaching the palace of wisdom; from his way of knowing, which is not exhausted in biorhythms, probabilistic models, primitive genomes, the uroborus eating its own tail, artificial neurons, phase transitions, complex systems, the Aztec code, surgical operations with robots, automata, remote medicine or Boolean networks.

I met Jacques in the IMAG tower of the University of Grenoble some forty years ago. I was starting my doctoral thesis and he, already a physician, had a significant advance in his State Thesis in Mathematics. Every week we met in a working group, led by François Robert (my thesis director), dedicated to Discrete Iterations-what today we could assimilate to Automata Networks. We were few, less than a dozen. Apart from François and Jacques, two common friends were also present: Michel Cosnard, future president of INRIA and Maurice Tchuente, later Minister of Education of Cameroon.

Eric Goles

eric.chacc@uai.cl

1 Facultad de Ingeniería y Ciencias, Universidad Adolfo Ibañez, Diagonal Las Torres, 2640 Peñalolen, Santiago, Chile
Since then my affection and admiration for Jacques began to develop. Was there any other possibility? His impressive scientific and humanistic culture, his overflowing enthusiasm, accompanied by everything of exceptional human quality and generosity, captivated me immediately. So, it was very easy for me to establish a friendship and complicity with Jacques that would culminate, a few years later, partly at his suggestion, with my joining the CNRS. Since then we have collaborated in various research projects, trained Chilean and French doctors, shared some sorrows, many joys and, perhaps most importantly, a conversation held over time; sometimes in Paris, sometimes in Grenoble or, here, in Santiago, at least once a year, often in the mythical National Bar.

And, well, there we were, just yesterday I would say, shared a cazuela, speaking of science, of the human and the divine, of what was and what will come, as if time did not pass as if this conversation were another and the same and, in truth, passing to the first person, neither what you tell me nor what I tell you has already happened, because the point is, my friend, that you have always managed to make all this happen in eternity.

\section{Conversaciones en el Bar Nacional}

La última vez que vino Jacques Demongeot a Santiago, hace apenas un par de meses, mientras comíamos una cazuela en el Bar Nacional, me comentó que una de sus tías había sido, en Roma, la madre superiora de una congregación cuyo nombre no recuerdo. Claro, agregó, era católico por familia y, por si acaso, siempre andaba con alguna reliquia. Antes que yo, supuestamente descreído (no lo estaba), le pidiera alguna prueba, sacó de la mochila una cajita con huesos santos.

La mayoría, aquellos que apenas lo conocen, luego de esta puesta en escena, terminarían rápidamente de comer y "si te he visto no me acuerdo". Pero, no yo, ni ninguno de los privilegiados que nos hemos dejado envolver por la magia de la maravillosa (y escasísima) persona que es: entre la alegre demasía de Rabelais y las aceradas certidumbres de Descartes. Y siempre desde la verdad, con el 
exceso necesario para, como afirmaba el poeta William Blake, alcanzar el palacio de la sabiduría; de su modo de saber, que no se agota en biorritmos, modelos probabilistas, genomas primitivos, el uróboros mordiéndose la cola, neuronas artificiales, transiciones de fase, sistemas complejos, el código azteca, operaciones quirúrgicas con robots, autómatas, medicina a distancia o redes booleanas.

Conocí a Jacques en la torre IMAG de la Universidad de Grenoble, hace unos cuarenta años. Yo comenzaba la tesis doctoral y él, ya médico, tenía un significativo avance en su Tesis de Estado en Matemática. Nos encontrábamos todas las semanas en un grupo de trabajo, liderado por François Robert (mi director de tesis), dedicado a las Iteraciones Discretas-lo que hoy podríamos asimilar a Redes de Autómatas. Éramos pocos, menos de una docena. Aparte de François y de Jacques, también asistían dos amigos comunes: Michel Cosnard, futuro presidente del INRIA y Maurice Tchuente, posteriormente Ministro de Educación de Camerún.

Desde ese entonces comenzó a fraguarse mi cariño y admiración por Jacques. ¿'Y cómo no? $\mathrm{Su}$ impresionante cultura científica y humanista, su entusiasmo desbordante, acompañado todo aquello de una calidad humana y generosidad excepcional, me cautivaron de inmediato. De modo que fue muy fácil para mi establecer una amistad y complicidad con Jacques que culminaría, pocos años más tarde, en parte por sugerencia suya, con mi incorporación al CNRS. Desde entonces hemos colaborado en diversas investigaciones, formado doctores chilenos y franceses, compartido algunas penas, muchísimas alegrías y, quizás lo más importante, una conversación sostenida en el tiempo; a veces en París, otras en Grenoble o, aquí, en Santiago, al menos una vez al año, a menudo en este algo mítico Bar Nacional.

Y, bueno, ahí estábamos, nomás ayer diría, cazuela de por medio, hablando de ciencia, de lo humano y lo divino, de lo que fue y de lo que vendrá, como si no pasara el tiempo como si esta conversación fuese otra y la misma y, en verdad, ni lo que me dices ni lo que te digo ya ocurrió, porque el asunto es, amigo mío, que siempre te has dado maña para que todo esto ocurra en la eternidad. 